\title{
ON THE GENERAL THEORY OF MULTIPLE CONTINGENCY WTTH SPECIAL REFERENCE TO PARTIAL CONTINGENCY.
}

\author{
BY KARL PEARSON, F.R.S.
}

(1) Let there be $l$ variates or characteristics $A, B, C, \ldots L$, each of these variates or characteristics being, subdivided into categories $A_{1}, A_{2}, \ldots A_{0}, B_{1}, B_{2}, \ldots B_{1}$, $C_{1}, C_{2}, \ldots C_{\gamma}, \ldots L_{1}, L_{2}, \ldots L_{\lambda}$, where $a, \beta, \gamma, \ldots \lambda$ are arbitrary numbers. Then if $N$ be the total population, and $n_{a_{1}}, n_{a_{1}}, \ldots$ the number of individuals in the 1-categories; $n_{b_{1}}, n_{b_{1}}, \ldots n_{b_{p}}$ those in the $B$-categories and 80 on, we have relations

$$
S_{1}^{*}\left(n_{a_{s}}\right)=S_{1}{ }^{\beta}\left(n_{b_{p}}\right)=S_{1}^{\gamma}\left(n_{c_{w}}\right)=\ldots=N \text {. }
$$

Further, if there be no relationship whatever between the variates or characteristics, we should anticipate that the frequency of the group $A_{*}, B_{0}, C_{w}, \ldots L_{\psi}$ in a sample of $\boldsymbol{M}$ would on the average be

$$
M \cdot \frac{n_{a_{n}}}{N} \cdot \frac{n_{b_{p}}}{N} \cdot \frac{n_{c_{b}}}{N} \cdots \frac{n_{L_{t}}}{N} \cdot
$$

Actaally we find in the sample $\boldsymbol{M}$ the number $m_{m o n}$, and the problem arises whether the system represented by $m_{\text {rom.... }}$ is so improbable that in the selected population $M$ the characteristics $A, B, C, \ldots L$ cannot be considered independent, i.e. $M$ is really not a random sample from the supposed population $N$. Clearly the answer to this problem has already been given. We have to find the value of $x^{2}$ :

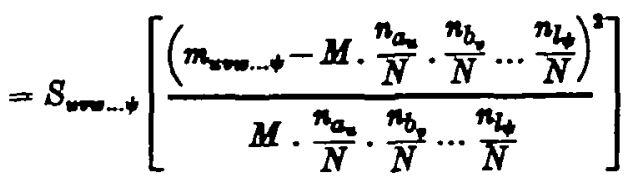

snd spply the tsbles for "goodness of fit." Of course in many cases the sampled population is not known and accordingly we can only put for $\frac{n_{a_{4}}}{N}, \frac{n_{b_{0}}}{N}, \ldots \frac{n_{L_{4}}}{N}$ the values given by the sample itself, i.e. $\frac{m_{a_{2}}}{M}, \frac{m_{b_{2}}}{M}, \ldots \frac{m_{L}}{M}$, and test from this substitution the degree of divergence from independence. If we take the mesn value of $\chi^{3}$, i.e. $\phi^{2}=\chi^{2} / M, \phi^{2}$ is termed the mean square contingency, and $C_{2}-\sqrt{\phi^{2} /\left(1+\phi^{2}\right)}$ 


\section{On the Theories of Multiple and Partial Contingency}

gives a measure of the divergence from independence*. This is a multiple contingency coefficient.

Another case not infrequently arises; the popalation $N$ hss the characteristics $A, B, C, \ldots$ not independent, but related ; the cell $u v r o \ldots \psi$ contains $n_{\text {wow... }}$ and the question arises how far it is safe to consider the population $M$ as a sample of this population. In this case $\dagger$

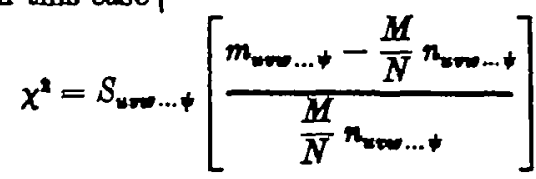

In both these cases we have the relation

$$
S_{\text {vir.... }}\left(m_{\text {wow ... }}\right)=\boldsymbol{M}
$$

and accordingly the number of cell frequencies is one more then the number of independent variates. Thus in using the tables $\ddagger$ of "goodness of fit" the $n$ ' of the argument is afy ... $\lambda$, but the value of $P$, the probability, has actually been determined from $n^{\prime}-1$.

(2) Now there are a number of cases in which not only do the cell-contents of the sample obey the linear relation (iii), but also other linear relations are imposed on the cell-contents. In the most general case we can suppose $q$ linear relations between the cell-contents $m_{w+\ldots} \ldots$, and obtain the probability $P$ corresponding to a value of $\chi^{2}$ limited by these $q$ relations. The theory of sampling, when such conditions are introduced, I term the theory of partial contingency. The reason for this terminology will be clearer as we develop the theory.

As far as we are concerned at present, it is of no importance whether we are dealing with one or other of our two cases, i.e. whether we are questioning the possibility of our material being a sample from a population with independent $A, B, C, \ldots L$ characteristics, i.e. determining a coefficient of mean squared contingency, or are investigating the passibility of its being a probable sample from a population with any associations between these characteristics. We can accordingly write $x^{2}$ in the form

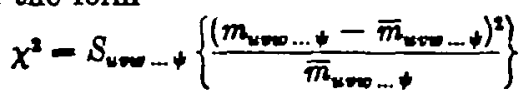

or for convenience we may even drop the descriptive subscripts and, numbering the cells in some sequence $1,2,3, \ldots s, \ldots(\alpha \beta \gamma \ldots \lambda)$, write

$$
\chi^{2}=S, \frac{\left(m_{s}-\bar{m}_{s}\right)^{2}}{\bar{m}_{s}}
$$

- If the charactaristics mag bo asomed to be continuous variates, certein corrections for mite of grouping can be mado. Thero is also a correction due to the necesurily positivo value of $\phi^{2}$. These corrections, which have been for some time in ase, will be considered ebowhere.

+ Wo must in this cave of courne sctarlly know the value of mess..., it cannot be jodged from the ample.

I For discustion of the deduction of $P$ from $\chi^{2}: s 00$ Phil Hag. Vol L p. 157 (July 1900); and for Tables: soo Tables for Statiaticiases and Biometricians, Table XII (Cembridgo Univentity Proan). 
Here $\bar{m}_{10 . \ldots}-\bar{m}_{a}$ will be either

as the case may be.

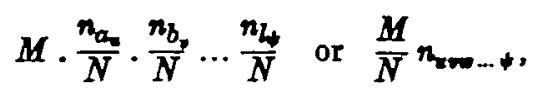

Now we shall find it convenient to write

and thus

$$
X_{\mathrm{s}}=\left(m_{\mathrm{s}}-\bar{m}_{\mathrm{s}}\right) / \sqrt{\overline{\boldsymbol{m}}_{\mathrm{s}}}
$$

$$
x^{2}=S_{s}\left(X_{2}^{2}\right)
$$

Further, if our $q$ linear equations be of type

$$
h_{t 1} m_{1}+h_{t 2} m_{2}+\ldots+h_{t s} m_{s}+\ldots=H_{t}
$$

where $h$ and $B$ are constants and $t$ takes every value from 1 to $q$, we can write our conditions in the form

where

$$
k_{i 1} X_{1}+k_{t 2} X_{2}+\ldots+k_{t} X_{s}+\ldots=K_{t}
$$

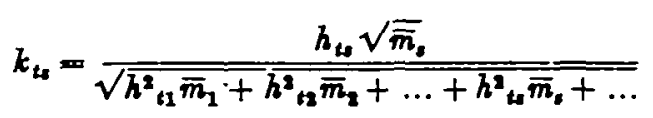

and

$$
K_{t}=\frac{B_{t}-h_{t 1} \bar{m}_{1}-h_{t 2} \bar{m}_{2}-\ldots-h_{t s} \bar{m}_{t}-\ldots}{\sqrt{h^{2}{ }_{11} \bar{m}_{1}+h^{2}{ }_{t 2} \bar{m}_{2}+\ldots+h^{2}{ }_{t s} \bar{m}_{t}+\ldots}}
$$

We shall speak of the first of (ix) as the prepared condition. Clearly it corresponds to a plane in $n$-dimensional space in which the constants $k_{t 1}, k_{t 2}, \ldots k_{t s}, \ldots$ are the direction-cosines and $K_{t}$ the perpendicular from the origin on the plane. It is convenient to use the notation

$$
k_{t_{1}} k_{t_{1}}+k_{t_{2}} k_{t^{\prime}}+\ldots+k_{t^{\prime}} k_{t^{\prime}}+\ldots=\cos \left(t^{\prime}\right)
$$

for $\left(t^{\prime}\right)$ is now the angle between the th and $t^{\prime}$ th planes. as

Assuming that the frequency surface with which we have to deal may be taken

$$
z=z_{0} e^{-\frac{1}{1} x^{2}}
$$

we may suppose before applying equations of condition (ix) that $K_{1}, K_{2}, \ldots K_{t}, \ldots K_{q}$ are variates and that we eliminate $X_{1}, X_{2}, \ldots X_{0}$, expressing our $\chi^{2}$ in terms of $K_{1}, K_{2}, \ldots K_{q}, X_{q+1}, X_{q+2}, \ldots X_{n}$. We shall have then

$$
z=z_{0} \text { expt. }-\frac{1}{1} \text { (quadratic function of these } n \text { new variables). }
$$

We now proceed to put $K_{1}, K_{2}, \ldots K_{q}$ constant, but lesve the other $n-q$ quantities to vary; we are therefore seeking the value of $\chi^{2}$ for certain variates constant. This is the essence of partial contingency, and the analogy in contingency to partial correlation.

(3) As a rule in partial contingency we do not seek to discuss $\chi^{2}$ when single cells of our multiple contingency solid are constant in frequency, although our theory covers that case. What we require usually is the value of $\chi^{2}$ when we make the contents of certain marginal total cells constant. For example, let us consider 


\section{On the Theories of Multiple and Partial Contingency}

a population of aniform sex and let there be three characteristics: (i) frequency of age groups, (ii) frequency of occupational categories and (iii) frequency of survival and of death, the latter classified by various special disease classes - the whole, say, representing the returns for one year of a large ares or country. We then require to determine, whether the like contingency solid for a sub-district, or for another population entirely, may be considered as significantly different from the sbove general population, i.e. we require to find the probability of its being a random sample of this general population. Now we may do this in the most universal manner, by assuming that not only survivals and deaths, but that age groups and occupations all have frequencies, which are random samples of the above general population. But this is not very often what we require; we admit that the age distribution is differentiated, we admit that the occupational frequencies are peculiar to the locality, and we ask whether, notwithstanding these differences, the death distribution is to be considered as a random sample.

In other words, we do not only fix the sise of our sample $M$; we fix all one face- that of age groups and occupational categories of our contingency solidand ask what is the distribution of asmples of $M$ taken from this solid, subject to the linear conditions that the totals of age-occupational categories are constant. For example, if $A$ be age and $B$ occupation, we make $n_{a_{\alpha} b}$ constant for all values of $u$ and $v$, but

$$
n_{a_{v} b_{v}}=n_{a_{v} b_{v} c_{1}}+n_{a_{v} b_{v} c_{1}}+n_{a_{v} b_{v} c_{3}}+\ldots,
$$

where $n_{a_{w} b_{n} c_{p}}$ is the frequency of the urs cell, $c$ denoting the category $C$ of type of death and survival.

Now clearly in making this investigation we shall be studying the mean square contingency and the resulting probability of a partial sample-a sample of survival and death-type in a population of constant age groups and occupstional classes. Again, we might treat a population as a sample with only constant age groups or only constant occupational frequencies and again investigate its probsbility as a sample with regard to deaths and occupations or with regard to deaths and ago groups respectively. These would be partial contingencies of the order a or $\beta$, while the previous partial contingency was of the order $\alpha+\beta$, $\alpha$ being the number of categories in $A$ (i.e. age groups) and $\beta$ being the number in $B$ (i.e. mortality and survival classes).

(4) Now the value of $\chi^{2}$ given above, and of the frequency surface ( $x$ ), was discussed by me in the year 1900 * from the general normal frequency surface by evalustion of determinants. The demonstration depends on two hypotheses:

(i) The approach of the binomial $M(p+q)^{n}$ to the normal curve

$$
y=-\frac{M}{\sqrt{2 \pi} \sqrt{n p q}} e^{-\frac{x^{2}}{2 n p q} .}
$$

We know that this is true if $n$ be considerable and neither $p$ nor $q$ very small.

- Phil Hag. Vol L. p. 157, 1900. I have recently given s moro elementery proof with the probablo arror of $P$ : 800 Pbil Mag. April, 1016. 
The hypothesis is justified therefore if no cell be taken 80 small that its contents are very small compared with the size of the sample.

(ii) That the sampling takes place out of a population indefinitely greater than the sample. If this be not true, then the distribution of frequency of any given cell for a series of samples follows not a binomial but a hypergeometrical series. The necessary modifications in the formulse are not very substantial and have ben discussed elsewhere*

Supposing the above two conditions to be fulfilled, then in true random samples the mean of a cell frequency will be

$$
\bar{m}_{\mathbf{s}}=\boldsymbol{M} \frac{\boldsymbol{n}_{\mathbf{s}}}{\bar{N}}
$$

where $M$ is size of sample, $n$, the contents of the sth cell in the sampled population $N$, and condition (i) amounts to saying that no cell is to be chosen so that $n_{2} / N$ is indefinitely small.

Further, the frequency of the sth cell will follow the binomial

$$
\left\{\frac{n_{s}}{N}+\left(1-\frac{n_{s}}{N}\right)\right\}^{*} \text {. }
$$

and thas have a standard deviation given by

$$
\sigma_{-\infty}=M \frac{n_{3}}{N}\left(1-\frac{n_{3}}{N}\right)
$$

Lastly the correlation $r_{z}$ between deviations in the sth and $s^{\prime}$ th cells is given by

$$
\sigma_{m,} \sigma_{n^{\prime}} \tau_{z^{\prime}}=-M \frac{n_{s}}{N} \frac{n_{s}}{N}
$$

(5) The following deduction of the value of $\chi^{2}$ is a variant from my Phi. Mag. proof. I owe the suggestion of it to $\mathbf{M r}$ H. E. Soper, although I have deviated somowhat from his track. Let an indefinitely large population $N$ consist of the classes $C_{0}, C_{1}, \ldots C_{1}$ in the quantities $n_{0}, n_{1}, n_{2}, \ldots n_{2}$ respectively. Then $p_{1}=n_{2} / N=$ chance of drawing a member of the class $C_{n}$, and the standard deviation of the distribution of frequency in samples of $M$ drawn from the population will in this class $C_{\mathrm{a}}$ be as above

$$
\sigma_{s}=\sqrt{\bar{M} p_{t}\left(1-p_{s}\right)}
$$

Further, the mean of samples for this class will be $\boldsymbol{M p}_{\mathbf{z}}$ by (rii).

In the next place the correlation between deviations from the means in classes $C_{\text {a }}$ and $C_{t}$ will be in our present notation

$$
\begin{aligned}
& \sigma_{s} \sigma_{t} \tau_{a^{\prime}}=-M n_{s} n_{s^{\prime}} / N^{2} \\
& (\text { (xiv) })^{\text {bet, }}
\end{aligned}
$$

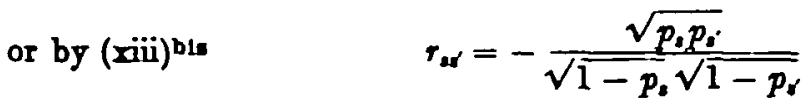

- Phil Kag. p. 239, 1899 and Biometrika, VoL v. p. 174 


\section{On the Theories of Multiple and Partial Contingency}

The distribution of frequency of the different classes in these samples of $M$ will be given by the terms of the multinomial

the general term being

$$
\left(p_{0} C_{0}+p_{1} C_{1}+p_{2} C_{2}+\ldots+p_{1} C_{1}\right)^{y}
$$

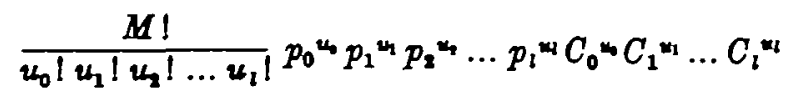

where $C_{0}, C_{1}, \ldots C_{2}$ are only logical symbols to denote that this general term is the frequency of the group, where the class $C_{\text {a }}$ occurs $u_{8}$, times in the sample. Clearly $u_{0}+u_{1}+\ldots+u_{t}=\boldsymbol{M}$.

But (xvi) may be put into the form of a binomial, it is

$$
=\sum_{m=0}^{m=M} \frac{M !}{m !(M-m) !}\left(p_{0} C_{0}\right)^{M-m}\left(p_{1} C_{1}+p_{2} C_{2}+\ldots+p_{l} C_{l}\right)^{m} \ldots \text { (xviii). }
$$

Let $\left(p_{1}+p_{z}+\ldots+p_{t}\right)=\lambda$, then the $m$ th term of the above series mag be read as

where

$$
\frac{M !}{m !(\boldsymbol{M}-m) !}\left(p_{0} C_{0}\right)^{M-m} \lambda^{m}\left(p_{1}^{\prime} C_{1}+p_{2}^{\prime} C_{2}+\ldots+p_{2}{ }^{\prime} C_{2}\right)=\ldots . .(\mathrm{xix}),
$$

and

$$
p_{1}^{\prime}+p_{2}^{\prime}+\ldots+p_{i}^{\prime}=1 \text {. }
$$

$$
\frac{p_{1}}{p_{1}^{\prime}}=\frac{p_{2}}{p_{2}^{\prime}}=\frac{p_{2}}{p_{3}^{\prime}}=\ldots=\frac{p_{l}}{p_{l}^{\prime}}=\lambda=1-p_{0}
$$

Now it is clear that the factor

$$
\left(p_{1}^{\prime} C_{1}+p_{2}^{\prime} C_{2}+\ldots+p_{l}^{\prime} C_{t}\right)^{m}
$$

give the frequency distribation of samples of $m$ drawn from a population of indefinitely large size of which the proportions of the classes $C_{1}, C_{2}, \ldots C_{1}$ are $p_{1}{ }^{\prime}, p_{2}{ }^{\prime}, \ldots p_{l}^{\prime}$ and in which no class $C_{0}$ occurs. But by $(x x)$ these proportions are the same as in the original population which contains $C_{0}$.

Hence if we take samples of $M$ from an indefinitely large population with classes $C_{0}, C_{1}, C_{x}, \ldots C_{1}$, those that contain $m$ of the classes $C_{1}, C_{2}, \ldots C_{t}$ will be distributed in the same proportions as if we had extracted $m$ from an indefinitely large popalation consisting only of those $l$ classes in the same proportions.

Now thus far the nature of the class $C_{0}$ is at our choice. In the original population $N$ it appears with the total frequency $n_{0}$. Let $n_{0}+n^{\prime}=N$, and suppose $n_{0}$ is indefinitely greater than $n^{\prime}$, then $p_{0}$ will be indefinitely greater than $p_{1}, p_{2}, \ldots p_{n}$. It follows that $p_{0}$ if $s$ be not eero is very small compared to unity, because

$$
p_{0}+p_{1}+p_{2}+\ldots+p_{n}=1 \text {. }
$$

Hence in such a system from (ziii) ${ }^{\text {bls, }}$, if $s$ be not zero,

$$
\sigma_{s}=\sqrt{\boldsymbol{M p} p_{2}}
$$

and from (xv) to the same degree of approrimation $r_{a t}=0$. That is to say, if $p_{0}$ be large in taking samples of size $\boldsymbol{M}$ from an indefinitely large population, there will be no correlation in deviations in the frequency of the classes $C_{1}, \ldots C_{2}$. 
On the other hand $r_{s 0}$ is not zero, bat equals by (zv)

$$
-\sqrt{p_{s} p_{0}} \sqrt{1-p_{0}}=-\sqrt{p_{s}} / \sqrt{1-p_{0}}
$$

to the same degree of approximation. Hence by $(\mathrm{x} x)$

$$
r_{\infty 0}=-\sqrt{p_{s}^{\prime}}
$$

But if we form the partial correlation of deviations in classes $C_{\text {a }}$ and $C_{t}$ for constant frequency of number in $C_{0}$, we have

or

$$
\begin{aligned}
& \sigma_{11}=\left(r_{A t}-r_{20} r_{t 0}\right) / \sqrt{\left(1-r_{20}{ }^{2}\right)\left(1-r_{t 0}{ }^{2}\right)} \\
& =\left(0-\sqrt{p_{t}^{\prime}} \sqrt{\left.p_{t}^{\prime}\right)} / \sqrt{\left(1-p_{t}^{\prime}\right)\left(1-p_{t}^{\prime}\right)}\right. \text {, } \\
& \sigma_{a t}=-\frac{\sqrt{p_{i}^{\prime} p_{i}^{\prime}}}{\sqrt{1-p_{t}^{\prime}} \sqrt{1-p_{t}^{\prime}}}
\end{aligned}
$$

which agres with (xv), the classes being now reduced by anity. Further, the reduced standard deviation must now be

$$
\begin{aligned}
\sigma_{0 \sigma_{s}} & =\sigma_{s} \sqrt{1-r_{0 a}^{2}} \\
& =\sigma_{s} \sqrt{1-p_{s}^{\prime}} \\
& =\sqrt{M p_{s}\left(1-p_{a}^{\prime}\right)} \\
& =\sqrt{M \lambda p_{s}^{\prime}\left(1-p_{s}^{\prime}\right)}
\end{aligned}
$$

Now take the mean value $\bar{x}_{8}$ of $x$, the frequency in class $C$, for a constant $x_{0}$ or for constant frequency in class $C_{0}$; in our case, if the sample is to be $m$ this will be $\boldsymbol{M}-\boldsymbol{m}$, we have

or

$$
\begin{aligned}
\bar{x}_{s}-\bar{x}_{z} & =r_{0 s} \frac{\sigma_{s}}{\sigma_{0}}\left(x_{0}-\bar{x}_{0}\right), \\
\bar{x}_{s}-M p_{s} & =\frac{-\sqrt{p_{s}^{\prime}} \sqrt{M p_{s}}}{\sqrt{\overline{M p_{0}}\left(1-p_{0}\right)}}\left(M-m-M p_{0}\right), \\
\bar{x}_{s} & =p_{s}\left\{M-\frac{1}{1-p_{0}}\left[M\left(1-p_{0}\right)-m\right]\right\} \\
\sigma_{s} & =\frac{m p_{s}}{1-p_{0}}=m \eta_{s}^{\prime} \ldots \ldots \ldots \ldots \ldots \ldots \ldots \ldots \ldots \ldots \ldots \ldots \ldots
\end{aligned}
$$

The partial values ${ }_{0} \bar{x}_{z}, o_{t s}$ of the means and correlations of classes for constant number in class $C_{0}$ are given by (xxii) and (xxv), and are what we might anticipate. But (xखiv) should be $\sqrt{m p_{s}^{\prime}\left(1-p_{s}^{\prime}\right)}$. It is accordingly needful to take

$$
\lambda=m / \boldsymbol{M} \text {, }
$$

or

$$
p_{0}=1-m / M
$$

These resulto have been reached on the assumption that $p_{0}$ is very large as compared with $p_{1}, p_{2}, \ldots p_{\mathrm{n}}$. It follows accordingly that the sample $M$ must be large as compared with $m$, and further the sum of the classes $C_{1}, C_{2}, \ldots C_{2}$ must 


\section{On the Theories of Multiple and Partial Contingency}

be to that of the class $C_{0}$ in the total population in the ratio of the partial asmple $m$ to the total sample $M$. Without this condition it is not possible to replace $M p$, by $m p_{a}^{\prime}$. Assuming these conditions to be satisfied, then samples of the size $m$ in classes $C_{1}, C_{2}, \ldots C_{1}$ picked out of very large samples of $\boldsymbol{H}$ will reproduce the same distribution of frequencies in those classes as samples of $m$ picked out of an indefinitely large population with the same relative frequencies in those classes.

But in the case of samples of $M$, the deviations have their correlations zero for the classes $C_{1}, C_{2}, \ldots C_{n}$, or they will be approximately distributed by the product of their independent probsbilities. The standard deviation being $\sqrt{m p_{s}^{\prime}}$ and the mean $m p^{\prime}$, we see that the frequency distribution would really follow a Poisson's binomial limit, but as shown by $\mathrm{L}$. Whitaker* this binomial limit is approximately Grussian with fairly low values of mpa'; see the Diagrams for $m p s^{\prime}-10$ and $=30$ in the plate of her memoir. We may accordingly therefore take the distribution of the frequency in the sth class or cell to be given by

$$
z_{s}=\frac{1}{\sqrt{2 \pi} \sqrt{\bar{m}_{z}}} e^{-\frac{1}{2} \frac{\left(m_{3}-\overline{\left.m_{2}\right)^{2}}\right.}{\bar{m}_{i}}}
$$

and the general distribution to be

where

$$
\left.\begin{array}{rl}
z & =\left(\frac{1}{\sqrt{2 \pi}}\right)^{l} \frac{1}{\sqrt{m^{2} p_{1}^{\prime} p_{2}^{\prime} \cdots p_{l}^{\prime}}} e^{-1 x^{a}} \\
X^{2} & =S_{1}\left\{\frac{\left(m_{s}-\overline{\left.m_{s}\right)^{2}}\right.}{\overline{m_{d}}}\right\}
\end{array}\right\}
$$

Here, if the size of the sample only be fixed, we shall have

$$
S\left(m_{\mathfrak{s}}\right)=m=m S\left(p_{s}^{\prime}\right)=S\left(\bar{m}_{s}\right) \text {, }
$$

or

$$
S\left(m_{1}-\bar{m}_{\jmath}\right)=0 \text {. }
$$

If we take

$$
X_{1}=\frac{m_{0}-\bar{m}_{1}}{\sqrt{\bar{m}_{1}}},
$$

we have:

$$
x^{2}-X_{1}^{2}+X_{1}^{2}+\ldots+X_{2}^{2}
$$

subject to the condition:

$$
\sqrt{\bar{m}_{1}} X_{1}+\sqrt{\bar{m}_{2}} X_{1}+\ldots+\sqrt{\bar{m}_{2}} X_{1}=0
$$

It is clear that $\chi^{2}$ equal to a constant gives a sphere in $l$-fold space, and thet $(\bar{x})$ is a plane passing through its centre, and therefore cutting the sphere in $l$-fold space in a sphere of the same radius in $(l-1)$-fold space. Hence if we desire to find the volume of the frequency surface (xrviii) which lies outaide a value of $x=x_{0}$ subject to the condition ( $\left.x a x\right)$, all we have to do is to transfer to polar

- Biometriba, Vol. I p. 38. If $\bar{\beta}_{1}=2 n p_{1}^{\prime}$ bo the mean, $\beta_{1}=1 / \overline{m_{1}}=\beta_{2}-3$, wo thet $\beta_{1}=-03, \beta_{2}=3-03$ alroedy for $\overline{x_{1}}=33$. 
coordinstes and integrate the value of $z$ for the $(l-1)$-fold surface beyond the value $\chi_{0}^{*}$. Accordingly

$$
P=\frac{\int_{x_{0}}^{\infty} e^{-1 x^{2}} x^{l-2} d x}{\int_{0}^{\infty} e^{-1 x^{2}} x^{l-2} d x} \ldots \ldots \ldots \ldots \ldots \ldots \ldots \ldots \text { (roxi) }
$$

is the chance of a sample occurring with as grest or greater devistion as the $\chi_{0}$ sample from the general population. This is the expression from which the Tables of "Goodness of Fit" were calculated, the arguments being $\chi_{0}{ }^{2}$ and $l$, i.e. the value of $x^{2}$ for the sample and the total number of categories in the sample. Thus far there is only difference of method of deduction, not of results.

(6) We now propose to replace condition (sx) by a series of $q$ linear equations of form (viii). These in the case of sampling will, if the sire of the sample be fixed, either directly or indirectly involve (oxx).

The type of these equations in their prepared form is

$$
k_{t i} X_{1}+k_{t 2} X_{2}+\ldots+k_{t} X_{t}+\ldots-K_{t} .
$$

Each such plane will intersect

$$
X^{2}=X_{1}^{2}+X_{2}^{2}+\ldots+X_{2}^{2}+\ldots
$$

in a sphere of lower order. For example, if there be $n$ variates $X$, the first plane gives a sphere of the $(n-1)$ th order, this will be intersected by the second plane in a sphere of the $(n-2)$ th order, so that ultimately we find ourselves reduced to a sphere of the $(n-q)$ th order, by the intersection of the qth plane. If $K_{1}$,

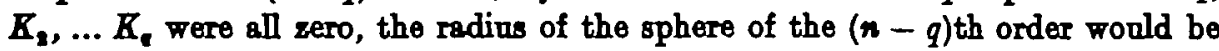
the same, i.e. $\chi^{2}$ as the radius of the sphere of the $n$th order. But since these quantities are usually not zero we have to determine the radius of this sphere. The centre of this sphere mnst lie in every one of the $q$ planes of the nth order, and accordingly on the plane of order $n-(q-1)$ in which they intersect. But the centre of the sphere of $n-q$ order is where the perpendicular, $K$, from the origin meets this plane of the $(n-\overline{q-1})$ th order, and the radins $x^{\prime}$ of the ephere of the $(n-q)$ th order is given by $\chi^{\prime 2}=\chi^{2}-K^{2}$. To determine $\chi^{\prime}$ we must find $P$.

Now $P$ will be the minimum distance from the origin to the plane of the $(n-\overline{q-1}$ )th order in which the $q$ planes intersect. In other words to find $P$ we must make

$$
D^{2}=X_{1}^{2}+X_{2}^{2}+\ldots+X_{1}^{2}+\ldots+X_{w}^{2}
$$

a minimum subject to the $q$ conditions of type

$$
k_{t 1} X_{1}+k_{t 2} X_{2}+\ldots+k_{t s} X_{t}+\ldots+k_{t w} X_{w}=K_{t} \text {, }
$$

where $\quad k_{u^{2}}+k_{t z}^{2}+\ldots+k_{t u}^{2}+\ldots+k_{t w}^{2}=1$.

Using the method of indeterminste multipliers we find $n$ equations of the form

$$
\begin{gathered}
X_{1}+\lambda_{1} k_{10}+\lambda_{2} k_{2}+\ldots+\lambda_{1} k_{m}=0 . \\
\text { •Pki Mag. Vol L p. } 168 .
\end{gathered}
$$




\section{On the Theories of Multiple and Partial Contingency}

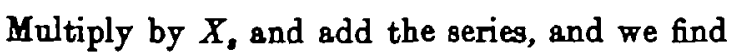

$$
-K^{2}=\lambda_{1} K_{1}+\lambda_{2} K_{2}+\ldots+\lambda_{1} K_{q}
$$

Multiply by $k_{1,}$ and add the series, and we find, by aid of $(x)$,

$$
-K_{1}=\lambda_{1}+\lambda_{2} \cos (12)+\lambda_{3} \cos (13)+\ldots+\lambda_{q} \cos (1 q) .
$$

Similarly :

$$
\begin{aligned}
& -K_{2}=\lambda_{1} \cos (21)+\lambda_{2}+\lambda_{3} \cos (23)+\ldots+\lambda_{q} \cos (2 q), \\
& -K_{t}=\lambda_{1} \cos (t 1)+\lambda_{2} \cos (t 2)+\ldots+\lambda_{t}+\ldots+\lambda_{1} \cos (t q), \\
& -B_{q}=\lambda_{1} \cos (q 1)+\lambda_{2} \cos (q 2)+\ldots+\ldots+\ldots+\lambda_{q} .
\end{aligned}
$$

These are $q$ equations to find $\lambda_{1}, \lambda_{2}, \ldots \lambda_{2}$, and we can then substitute in (xxxii) to find the required $P^{2}$.

Now consider the determinant

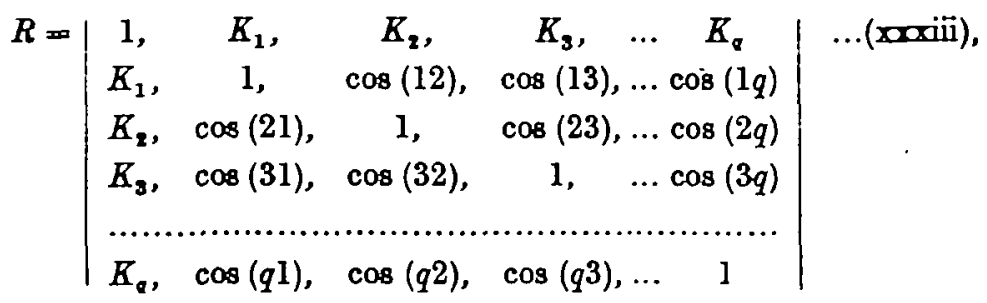

and let us call the first row and the first column the 0 row and 0 column, then clearly

$$
-\lambda_{t}=-R_{00} t / R_{00},
$$

where $R_{n}$ is the minor of the sth row and $t$ th column, and

$$
\begin{aligned}
-K^{2}=\frac{K_{1} R_{01}+K_{2} R_{02}+\ldots+K_{t} R_{0 t}+\ldots+K_{\mathrm{a}} R_{00}}{R_{00}}, \\
1-K^{2}=R / R_{000}, \\
K^{2}=1-R / R_{00} \ldots \ldots \ldots \ldots \ldots \ldots \ldots \ldots \ldots \ldots \ldots \ldots \ldots \ldots \ldots \text { (xxiv). }
\end{aligned}
$$

If we call $\Delta$ the minor $R_{00}$, we have:

$$
\begin{gathered}
R_{0 t}=-K_{1} \Delta_{1 t}-K_{2} \Delta_{2 t}-\ldots-K_{t} \Delta_{t t}-\ldots \\
K^{2}=S\left(K_{t}^{2} \frac{\Delta_{t t}}{\Delta}\right)+2 S\left(K_{t} K_{t} \frac{\Delta_{t t}}{\Delta}\right) \ldots \ldots
\end{gathered}
$$

From this we deduce that the probability of a sample which gives $\chi^{2}=\chi_{0}^{2}$ with $q$ linear conditions must be obtained from

$$
P=\frac{\int_{x_{0}^{2}-\Sigma^{2}}^{\infty} e^{-\frac{1}{2} x^{2}} \chi^{n-a-1} d x}{\int_{0}^{\infty} e^{-1 \chi^{2}} \chi^{n-a-1} d x}
$$


We must therefore in order to find $P$ enter the Tables of "Goodness of Fit" with $\chi^{2}=\chi_{0}^{2}-K^{2}$ and with $n^{\prime}=n-q+1^{*}$.

The reader who is familiar with the theory of the multiple total and partial correlation coefficients will note how closely anslogous the formulae (uriii) to (xoxv) are to results in that theory. The fundamental determinant of the correlation may be made to agree with $\Delta$, if we merely write $r_{2 \alpha^{\prime}}=\cos \left(2 s^{\prime}\right)$. In fact both theories really reduce to the discussion of the formulae of spherical trigonometry in multiple space.

(7) A simple illustration of the above formulae may be taken from the case of the distribution of mortality in two districts, where the problem is to ascertain the probability that the difference of mortality observed allowing for the frequency of the age groups could be due to random sampling.

Let the population sampled be represented by

(a)

$$
\begin{array}{c|c|c|c|c|c|c|c}
D_{1} & D_{2} & D_{3} & \ldots \ldots & D_{2} & \ldots \ldots & D_{4} & \Delta \\
L_{1} & L_{2} & L_{3} & \ldots \ldots & L_{3} & \ldots \ldots & L_{4} & \Lambda \\
\hline A_{1} & A_{1} & A_{3} & \ldots \ldots & A_{2} & \ldots \ldots & A_{4} & P
\end{array}
$$

where $D$, are the dead, $L$, the living and $A$, the exposed to risk in the sth age group, there being $u$ such groups in the total population $P$, of whom in the given period $\Delta$ die and $\Lambda$ survive.

Let the districts be represented by

and

$$
\begin{array}{l|l|l|l|l|l|l|l}
d_{1} & d_{2} & d_{3} & \ldots \ldots & d_{8} & \ldots \ldots & d_{v} & 8 \\
l_{1} & l_{2} & b_{3} & \ldots \ldots & l_{8} & \ldots \ldots & l_{*} & \lambda \\
\hline a_{1} & a_{2} & a_{3} & \ldots \ldots & a_{1} & \ldots \ldots & a_{*} & p
\end{array}
$$

$$
\begin{array}{c|c|c|c|c|c|c|c}
d_{1}^{\prime} & d_{2}^{\prime} & d_{3}^{\prime} & \ldots \ldots & d_{i}^{\prime} & \ldots \ldots & d_{*}^{\prime} & \delta^{\prime} \\
l_{1}^{\prime} & l_{2}^{\prime} & b_{3}^{\prime} & \ldots \ldots & l_{s}^{\prime} & \ldots \ldots & l_{*}^{\prime} & \lambda^{\prime} \\
\hline a_{1}^{\prime} & a_{2}^{\prime} & a_{3}^{\prime} & \ldots \ldots & a_{s}^{\prime} & \ldots \ldots & a_{z}^{\prime} & p^{\prime}
\end{array}
$$

respectively. Then the problem is to ascertain the probability that the last two distributions could both be samples of the same first population.

The general formula has been given by met; we have to evaluate

$$
x^{2}=S\left\{\frac{\left(\frac{f_{t}}{p}-\frac{f_{t}^{\prime}}{p^{\prime}}\right)^{2}}{\left(\frac{1}{p}+\frac{1}{p^{\prime}}\right) \frac{P_{t}}{P}}\right\},
$$

- The tables are constructed for $n^{\prime}-1$ independent variables; in our case thero aro $n-q$ such varisbles, bence $n^{\prime}-1=\pi-q$.

† Biometrika, Vol vin. p. 252. 
where $f_{i}$ must take the value of every frequency in the cells of $(\beta), f_{i}^{\prime}$ the corresponding cell frequency for $(\gamma)$, and $F_{t}$ that for $(a)$. We have to seek for $P$ under the argument $n^{\prime}=2 u$ in our tables, if we have no restrictions on our variates. Actually we have such restrictions, for we are going to seek the partial $\chi^{2}$ when we suppose the age groups in each sample to be constant. Now

Thus it follows that

$$
d_{z}+l_{s}=a_{s} \text { and } d_{s}^{\prime}+l_{s}^{\prime}=a_{a}^{\prime} \text {. }
$$

$$
\frac{d_{s}}{p}-\frac{d_{s}^{\prime}}{p^{\prime}}+\frac{l_{s}}{p}-\frac{l_{s}^{\prime}}{p^{\prime}}=\frac{a_{z}}{p}-\frac{a_{i}^{\prime}}{p^{\prime}}
$$

We have accordingly $u$ equations of condition or $n^{\prime}$ as argument will be reduced to $u+1$. Now we take

Thus

$$
\begin{aligned}
& X_{s}=\sqrt{\frac{p p^{\prime}}{p+p^{\prime}}} \frac{\left(\frac{d_{s}}{p}-\frac{d_{s}^{\prime}}{p^{\prime}}\right)}{\sqrt{D_{d} P}}, \\
& Y_{s}=\sqrt{\frac{p p^{\prime}}{p+p^{\prime}}} \frac{\left(\frac{l_{s}}{p}-\frac{l_{1}^{\prime}}{p^{\prime}}\right)}{\sqrt{L_{s} P}} .
\end{aligned}
$$

with $u$ conditions of form

or in the prepared form

$$
\sqrt{D_{2} P} X_{1}+\sqrt{L_{2}} P Y_{2}=\sqrt{\frac{p p^{\prime}}{p+p^{\prime}}}\left(\frac{a_{3}}{p}-\frac{a_{1}^{\prime}}{p^{\prime}}\right)
$$

$$
\sqrt{\frac{D_{i}}{A_{1}}} X_{s}+\sqrt{\frac{L_{a}}{A_{a}}} Y_{1}=\sqrt{\frac{p p^{\prime}}{p+p^{\prime}}} \frac{\left(\frac{a_{1}}{p}-\frac{a_{s}^{\prime}}{p^{\prime}}\right)}{\sqrt{\frac{A_{a}}{P}}} .
$$

Thus

$$
K_{i}=\sqrt{\frac{p p^{\prime}}{p+p^{\prime}}}\left(\frac{a_{t}}{p}-\frac{a_{s}^{\prime}}{p^{\prime}}\right) / \sqrt{\frac{A_{t}}{P}}
$$

Further, all the cosines like cos $\left(s s^{\prime}\right)$ are zero. for no equations of condition involve the same variates*. Thus

Accordingly

$$
\begin{aligned}
K^{2} & =K_{1}^{2}+K_{2}^{2}+\ldots+K_{*}^{2} \\
& =\frac{p p^{\prime}}{p+p^{\prime}} S_{2}=\frac{P}{A_{1}}\left(\frac{a_{1}}{p}-\frac{a_{a}^{\prime}}{p^{\prime}}\right)^{2}
\end{aligned}
$$

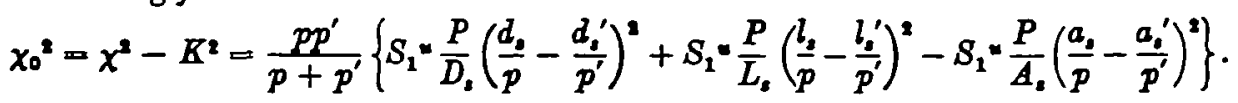

We shsll now substitute from the relation (ruvii), getting rid of $\frac{l_{*}}{p}-\frac{l_{s}^{\prime}}{p^{\prime}}$.

- Thin will necensarily be trae if our equations of condition refer to parallel row or columne, not if they refer to certain rows and columns. 
We find

$$
\begin{aligned}
& \chi_{0}^{2}=\frac{p p^{\prime}}{p+p^{\prime}}\left\{S_{1}{ }^{*} \frac{P A_{s}}{D_{s} L_{s}}\left(\frac{d_{s}}{p}-\frac{d_{s}^{\prime}}{p^{\prime}}\right)^{2}-\frac{2 P}{L_{s}}\left(\frac{d_{s}}{p}-\frac{d_{s}^{\prime}}{p^{\prime}}\right)\left(\frac{a_{s}}{p}-\frac{a_{s}^{\prime}}{p^{\prime}}\right)+\frac{P D_{s}}{A_{s} L_{s}}\left(\frac{a_{s}}{p}-\frac{a_{s}^{\prime}}{p^{\prime}}\right)^{2}\right\} \\
& =\frac{p p^{\prime}}{p+p^{\prime}} S_{1} *\left\{\frac{P A_{s}}{D_{s} L_{s}}\left[\frac{d_{s}}{p}-\frac{d_{s}^{\prime}}{p^{\prime}}-\frac{D_{s}}{d_{s}}\left(\frac{q_{s}}{p}-\frac{a_{s}^{\prime}}{p^{\prime}}\right)\right]^{2}\right\} \text {. } \\
& \text { Let } \frac{D_{s}}{A_{s}}=p_{s}, \frac{L_{1}}{I_{1}}=q_{s} \text {, then we may write } \\
& \chi_{0}^{2}=\frac{p p^{\prime}}{p+p^{\prime}}, S_{1} *\left\{\frac{P}{A_{s} p_{s} q_{s}}\left(\frac{d_{1}-p_{s} a_{s}}{p}-\frac{d_{s}^{\prime}-p_{s} a_{a}^{\prime}}{p^{\prime}}\right)^{2}\right\} .
\end{aligned}
$$

Now if we know the population sampled, we have only to insert the values of $P, A_{s}$, and $p_{s}, q_{s}$ to obtain the value of $\chi_{0}^{2}$. Bat if we do not, and this is usually the case, then our problem is: Are the two districts samples of the same unknown genersl population? For example, we might enquire whether the death distributions in Bradford and Leeds were, correcting for age, significantly different. It might be supposed that it would be correct to give $A_{s} / P, p_{s}$ and $q_{s}$, the values found for all England and Wales. But it.may be doubted whether this would be satisfactory. The populations of Bradford and Leeds might fairly be considered as asmples of a general population which is very far indeed from being that of all Fingland. Accordingly it seerns much more reasonable to suppose that they are samples of a population whose mortality characters will be best represented by the combination of those of the two districts themselves, or we take

$$
p_{s}=\frac{d_{s}+d_{s}^{\prime}}{a_{s}+a_{s}^{\prime}}, \quad \frac{A_{s}}{P}=\frac{a_{s}+a_{s}^{\prime}}{p+p^{\prime}} .
$$

Substituting the first of these relations we find

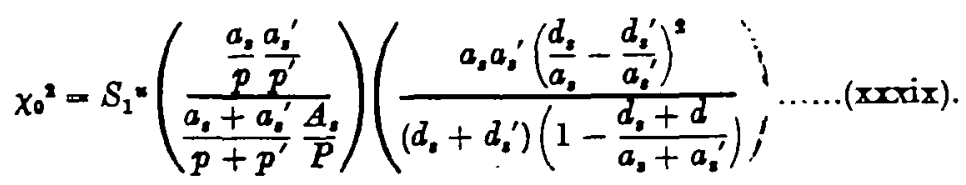

If we substitute the second relation, (xxxix) becomes

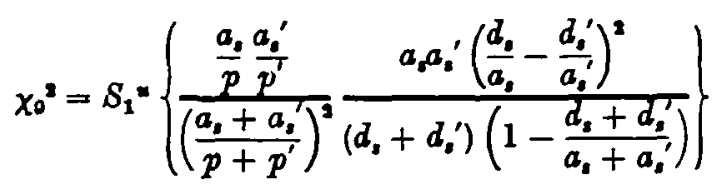

and this, I take it, is the best messure of aignificance in the difference of the death distributions allowing for age groups in the two districts.

It is clear that the first factor will in many cases differ but little from anity. For we should anticipate that approximately

$$
a_{1}=\frac{p}{P} A_{s,} \quad a_{1}^{\prime}=\frac{p^{\prime}}{P} A_{s}
$$




\section{On the Theories of Multiple and Partial Contingency}

Hence it would follow that

$$
\frac{a_{a}}{p} \frac{a_{s}^{\prime}}{p^{\prime}}=\left(\frac{A_{d}}{P}\right)^{2}=\left(\frac{a_{s}+a_{a}^{\prime}}{p+p^{\prime}}\right)^{2},
$$

or the first factor is approximately unity. The value of $\chi_{0}^{2}$ then becomes

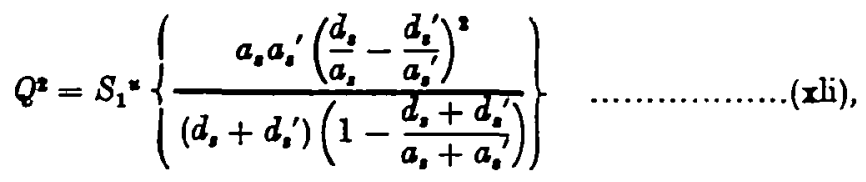

a quantity which I have shown elsewhere* has an important relation to the question of the significant difference of corrected death rates.

It is obvious that the present method is of wide generality and I propose to illustrate it later by considering whether the general death distributions of various national groups allowing for age distribution, occupation distribution and character of mortality are or are not significantly different.

Meanwhile (worix) provides us with a formula which ensbles us readily in the case of districts, special diseases or class groups to assert whether mortality experiences corrected for age distribution are or are not significantly different. As far as I can see (maxix) and its extensions to occupation groups provide the proper means of ascertaining whether the populations at risk in various insurance offices or friendly societies are or are not materially different in character. It should be a guide to the actuary also as to which classes of the population it is most desirable to cater for.

I have to thank my friends Mr H. E. Soper and Mr A. W. Young for suggestions and help at several points. In the following paper numerical illustrations of (zl) are provided. 